\title{
Alkylresorcinols (biomarkers of whole grain intake), cereal fibre intake and metabolic profile - results from a European study
}

\author{
Cecilie Kyrø ${ }^{1}$, Izabela Biskup ${ }^{2}$, Carl Brunius ${ }^{2}$, Birthe Lykke Riegels Thomsen ${ }^{1}$, Isabelle Romieu ${ }^{3}$, \\ Mazda Jenab ${ }^{3}$, Anne Kirstine Eriksen ${ }^{1}$, Bas Bueno-de-Mesquita ${ }^{4,5}$, Elio Riboli ${ }^{5}$, Yunxia Lu ${ }^{5,6}$, \\ Elisabete Weiderpass ${ }^{3}$, Krasimira Aleksandrova ${ }^{7}$, Anne Tjønneland ${ }^{1}$, Anja Olsen ${ }^{1}$ and \\ Rikard Landberg 2,8 \\ ${ }^{1}$ Danish Cancer Society Research Center, Copenhagen, Denmark, \\ ${ }^{2}$ Department of Biology and Biological Engineering, Food and Nutrition Science, Chalmers University of Technology, \\ Gothenburg, Sweden, \\ ${ }^{3}$ International Agency for Research on Cancer (WHO-IARC), Lyon, France, \\ ${ }^{4}$ National Institute for Public Health and the Environment (RIVM), Bilthoven, Netherlands, \\ ${ }^{5}$ School of Public Health, Imperial College London, London, United Kingdom, \\ ${ }^{6}$ Department of Population Health and Disease Prevention, Program in Public Health, University of California, Irvine, \\ USA, \\ ${ }^{7}$ German Institute of Human Nutrition Potsdam-Rehbruecke, Nuthetal, Germany and \\ ${ }^{8}$ Department of Public Health and Clinical Medicine, Umeå University, Umea, Sweden
}

\begin{abstract}
Background: High intakes of whole-grains and cereal fiber have been consistently associated with lower risk of cardiometabolic diseases in observational studies. Yet, improved understanding about the underlying mechanisms is needed. We hypothesized that cereal fiber and whole-grain are associated with beneficial metabolic marker profiles.

Aim: To investigate if cereal fiber intake, estimated by food frequency questionnaires and plasma total alkylresorcinols concentrations as well as the C17:0/C21:0-ratio in plasma as biomarkers of whole-grain wheat and rye intake or the relative whole-grain rye to wheat intake, respectively, were associated with metabolic biomarkers.

Design: A cross-sectional study conducted to investigate the associations between alkylresorcinols as biomarker of whole-grain wheat and rye intake, cereal fiber and selected metabolic biomarkers among 954 participants of the European Prospective Investigation into Cancer and Nutrition (EPIC) study. Cereal fiber intake was assessed by FFQ and whole grain wheat and rye were reflected by biomarkers analyzed in plasma samples, i.e. total alkylresorcinol (AR). Moreover, the ratio of two of the five measured alkylresorcinols (AR C17:0/C21:0 ratio) was used as an indicator of whole-grain source (wheat or rye). Metabolic biomarkers (HbA1c, C-peptide, IGFBP-1, IGFBP-2, total cholesterol, LDL, HDL, triglycerides, apolipoprotein A-I (apoA), apolipoprotein B (ApoB) and CRP) were measured in blood samples. All biomarkers were already measured for nested case-control studies of colorectal cancer matched based on sex, study center, age at blood collection, date and time of blood collection, fasting status. Women were further matched by menopausal status, phase of menstrual cycle, and use of oral contraceptives or hormone replacement therapy at time of blood collection. Multivariable linear regression analysis was used to investigate the relationship between exposure variables metabolic biomarkers adjusted for case-control status and common confounders.
\end{abstract}

Results: No associations were found between cereal fiber intake and the metabolic markers. However, whole-grain wheat and rye intake, reflected by total AR, was associated with a lower concentration of the inflammation marker CRP. The alkylresorcinol $\mathrm{C} 17: 0 / \mathrm{C} 21: 0$ ratio was not associated with any of the measured metabolic markers in this cohort.

Conclusion: Overall, we found no support for an association between cereal fibre intake, whole grain wheat and rye intake reflected by biomarkers and metabolic markers in the present cohort. One exception was the finding of an inverse association between whole grain biomarkers and CRP. Prospective studies or RCTs are warranted to confirm our findings.

\section{Conflict of Interest}

There is no conflict of interest 